\title{
Cardiovascular toxicities from systemic breast cancer therapy
}

\author{
Shuang Guo ${ }^{1}$ and Serena Wong ${ }^{2}$. \\ ${ }_{1}$ Rutgers Robert Wood Johnson Medical School, Rutgers, The State University of New Jersey, New Brunswick, NJ, USA \\ 2 Division of Medical Oncology, Rutgers Cancer Institute of New Jersey, Rutgers Robert Wood Johnson Medical School, Rutgers, The State University of New \\ Jersey, New Brunswick, NJ, USA
}

Edited by:

Sharad Goyal, Rutgers, The State

University of New Jersey, USA

\section{Reviewed by:}

Anastasia Pazaiti, emBIO Medical

Center, Greece

Paul Stephen Rava, University of

Massachusetts Memorial Medical

Center, USA

${ }^{*}$ Correspondence:

Serena Wong, Division of Medical

Oncology, Rutgers Cancer Institute of

New Jersey, Rutgers Robert Wood

Johnson Medical School, Rutgers,

The State University of New

Jersey, 195 Little Albany Street, New

Brunswick, NJ 08903, USA

e-mail:wongse@cinj.rutgers.edu
Cardiovascular toxicity is unfortunately a potential short- or long-term sequela of breast cancer therapy. Both conventional chemotherapeutic agents such as anthracyclines and newer targeted agents such as trastuzumab can cause varying degrees of cardiac dysfunction. Type I cardiac toxicity is dose-dependent and irreversible, whereas Type II is not dose-dependent and is generally reversible with cessation of the drug. In this review, we discuss what is currently known about the cardiovascular effects of systemic breast cancer treatments, with a focus on the putative mechanisms of toxicity, the role of biomarkers, and potential methods of preventing and minimizing cardiovascular complications.

\section{Keywords: cardiotoxicity, heart failure, chemotherapy, breast cancer, anthracycline, trastuzumab}

\section{INTRODUCTION}

Breast cancer remains the most common cancer among women. It is estimated that more than 3.1 million women with a history of invasive breast cancer are alive in the United States. By 2024, that number is estimated to increase to 3.9 million (1). Advances in breast cancer treatments have fortunately led to improvements in survival. However, with more women living longer, delayed toxicities of therapy have become more significant. Cardiovascular complications from therapy can occur to varying degrees and, in severe cases, can have devastating consequences.

Cardiotoxicity from breast cancer therapy is seen most commonly after treatment with anthracyclines and trastuzumab, at times necessitating discontinuation of otherwise effective treatment. Yet despite the large number of studies that have addressed the cardiotoxic effects from breast cancer therapy, few guidelines exist for the detection, monitoring, and management of patients with treatment-related cardiotoxicity.

Cardio-oncology has emerged as a new field that focuses on preserving cardiovascular health in cancer patients. Key areas of research include understanding the mechanisms of cardiac dysfunction, developing biomarkers for early detection, and instituting appropriate therapy for both the prevention and treatment of cardiac toxicity.

\section{ANTHRACYCLINE-INDUCED CARDIOTOXICITY}

The role of anthracyclines in the treatment of breast cancer is well-established $(2,3)$. However, the benefit of this class of drugs is often limited by the risk of myocardial damage which, in severe cases, can progress to symptomatic congestive heart failure (CHF). Anthracycline-induced cardiotoxicity can take on a variety of forms. Acute cardiotoxicity is relatively uncommon and is usually not life threatening. It can manifest as electrocardiogram changes, arrhythmias, and transient depression of myocardial contractility. These changes generally occur during intravenous administration and normally resolve on their own with discontinuation of the drug (4-6). Chronic anthracycline-induced cardiotoxicity, on the other hand, is more common and of much greater clinical concern. This type of cardiotoxicity is classified as Type I and is dose-related, progressive and irreversible.

Chronic cardiotoxicity typically presents within 1 year of treatment but late manifestations can occur up to 10 or more years following anthracycline therapy (7). Asymptomatic diastolic dysfunction may be an early finding in patients exposed to anthracycline therapy; this may progress to heart failure with preserved left ventricular ejection fraction (LVEF) and eventually to heart failure with reduced EF. Endomyocardial biopsies performed on patients exposed to anthracyclines have demonstrated ultrastructural changes such as vacuolization, myofibrillar disorganization, and myocyte necrosis, even in the absence of overt clinical symptoms $(8,9)$.

The dose-dependent relationship of anthracyclines and cardiotoxicity has been well-characterized since the late 1970s. In a retrospective analysis of 4018 patients with a variety of tumors who received doxorubicin, the number of patients who developed $\mathrm{CHF}$ was $3 \%$ at a cumulative doxorubicin dose of $400 \mathrm{mg} / \mathrm{m}^{2}, 7 \%$ at a cumulative dose of $550 \mathrm{mg} / \mathrm{m}^{2}$, and $18 \%$ at a dose of $700 \mathrm{mg} / \mathrm{m}^{2}$ (4). However, another analysis of doxorubicin-associated cardiotoxicity revealed an even higher incidence of $\mathrm{CHF}$ at $26 \%$ with a cumulative dose of $550 \mathrm{mg} / \mathrm{m}^{2}(10)$. These authors also found that CHF occurred at total cumulative doses of $<300 \mathrm{mg} / \mathrm{m}^{2}$, although 
this was relatively infrequent. Based on these observations, it is generally recommended that the cumulative dose of doxorubicin be limited to $400-450 \mathrm{mg} / \mathrm{m}^{2}$ in adults (see Table 1 ).

While cumulative dose remains the most significant risk factor for the development of cardiotoxicity, other risk factors include older age, radiation therapy, concomitant chemotherapy, and factors that may predispose to cardiovascular disease such as hypertension and diabetes $(4,10-13)$. However, there is great variability in patient susceptibility to cardiotoxicity. In the analysis by Von Hoff, five patients received over $1000 \mathrm{mg} / \mathrm{m}^{2}$ of doxorubicin and did not develop clinical cardiotoxicity, while others developed $\mathrm{CHF}$ at much lower cumulative doses (4). Genetic polymorphisms may account at least in part for some of the differences in susceptibility, although studies performed in the pediatric population have yielded conflicting results $(14,15)$.

\section{MECHANISM OF ANTHRACYCLINE-INDUCED CARDIOTOXICITY}

Cardiac myocytes have limited regenerative capabilities and thus are especially susceptible to irreversible damage. The mechanism by which anthracyclines induce cardiotoxicity has been a topic of great debate and multiple hypotheses have been proposed. Until recently the most widely accepted explanation has been the oxidative stress model whereby generation of reactive oxygen species (ROS) by redox cycling and iron-anthracycline complexes leads to myocyte damage. However, this hypothesis has been called into question given the failure of antioxidants such as vitamin E, coenzyme Q10, and $N$-acetylcysteine and iron chelators such a deferasirox to confer a cardioprotective effect in the clinical setting (16-19).

More recently, investigators from MD Anderson Center proposed an alternate explanation for anthracycline-induced cardiotoxicity via the topoisomerase (Top) 2 b enzyme (20). Anthracycline binding to Top2 is a well-established mechanism of cellular damage and antitumor activity. Top $2 \mathrm{a}$ is overexpressed in cancer cells but is absent in normal cells, whereas Top2b is expressed only in normal cells, including cardiac myocytes. Using a mouse knockout model, the researchers demonstrated that cardiomyocyte-specific deletion of Top $2 b$ conferred protection against doxorubicin-induced DNA damage, mitochondrial dysfunction and generation of ROS. In addition, mice lacking the Top2b gene in cardiac muscle did not develop progressive heart failure, whereas those with intact Top2 showed a decrease in EF after doxorubicin exposure. Based on these findings, the investigators are now evaluating the utility of a Top2 blood test in predicting sensitivity to doxorubicin-induced cardiac toxicity. If the Top2 hypothesis is confirmed, one potential alternative to avoid doxorubicin-induced cardiotoxicity could be to develop Top2aspecific anthracyclines that would have maximum antitumor activity without causing cardiotoxicity.

\section{CARDIOPROTECTANTS}

Several strategies have been used in an attempt to minimize anthracycline-induced cardiotoxicity. Dexrazoxane is a cardioprotective agent that is currently indicated for reducing the incidence and severity of cardiomyopathy associated with doxorubicin administration in patients with metastatic breast cancer, who have
Table 1 | Incidence of doxorubicin-induced CHF in the metastatic setting.

\begin{tabular}{|c|c|c|c|c|}
\hline Study & $\begin{array}{l}\text { Number of } \\
\text { patients } \\
\text { in analysis }\end{array}$ & Malignancy & $\begin{array}{l}\text { Overall } \\
\text { incidence } \\
\text { of CHF (\%) }\end{array}$ & $\begin{array}{l}\text { Incidence of CHF } \\
\text { based on } \\
\text { cumulative dose } \\
\text { of doxorubicin }\end{array}$ \\
\hline $\begin{array}{l}\text { Von Hoff } \\
\text { et al. (4) }\end{array}$ & 4018 & $\begin{array}{l}\text { Variety of } \\
\text { tumors }\end{array}$ & $2.2^{\mathrm{a}}$ & $\begin{array}{l}3 \% \text { at } 400 \mathrm{mg} / \mathrm{m}^{2} \\
7 \% \text { at } 550 \mathrm{mg} / \mathrm{m}^{2} \\
18 \% \text { at } 700 \mathrm{mg} / \mathrm{m}^{2}\end{array}$ \\
\hline $\begin{array}{l}\text { Swain } \\
\text { et al. (10) }\end{array}$ & 630 & $\begin{array}{l}\text { Metastatic } \\
\text { breast cancer } \\
\text { and small cell } \\
\text { lung cancer }\end{array}$ & $5.1^{\mathrm{b}}$ & $\begin{array}{l}5 \% \text { at } 400 \mathrm{mg} / \mathrm{m}^{2} \\
16 \% \text { at } 500 \mathrm{mg} / \mathrm{m}^{2} \\
26 \% \text { at } 550 \mathrm{mg} / \mathrm{m}^{2} \\
48 \% \text { at } 700 \mathrm{mg} / \mathrm{m}^{2}\end{array}$ \\
\hline
\end{tabular}

${ }^{a}$ Based on clinical signs and symptoms of $\mathrm{CHF}$.

${ }^{b}$ Protocol definition of CHF included two or more of the following: cardiomegaly on chest X-ray; basilar rales; S3 gallop; or paroxysmal nocturnal dyspnea, orthopnea, or significant dyspnea on exertion.

received a cumulative doxorubicin dose of $300 \mathrm{mg} / \mathrm{m}^{2}$ and who will continue to receive doxorubicin therapy to maintain tumor control. Multiple studies have demonstrated a cardioprotective effect when this agent is used during anthracycline treatment for advanced breast cancer (21-25). In all of these studies, patients who received dexrazoxane had a decreased incidence of $\mathrm{CHF}$ compared to those who did not receive the drug. Yet despite these consistent positive findings, the use of dexrazoxane has not been widely adopted. This is due in part to the suggestion from a single study that dexrazoxane may lower response rates, although there was no impact on time to progression (TTP) or overall survival (OS) seen in that trial (23). This finding has not been seen in other studies, and a meta-analysis of five randomized trials $(n=818)$ showed that there was no difference in response rates between control patients and patients receiving dexrazoxane (26).

The mechanism by which dexrazoxane is thought to confer cardioprotection has not been fully elucidated. By binding to the anthracycline-iron complex and removing iron, dexrazoxane may prevent free radical-induced lipid peroxidation of mitochondrial membranes and endoplasmic reticulum $(27,28)$. However, this theory of iron chelation does not fully explain the cardioprotective mechanism of the drug given the observation that deferasirox, an efficient iron-chelating agent that has been shown to enter myocytes and displace iron from the anthracycline-iron complex, is unable to protect myocytes from doxorubicin damage $(19,28)$. An alternate hypothesis involves the ability of dexrazoxane to prevent anthracycline binding to Top2. As discussed above, when doxorubicin binds to Top2b in cardiac myocytes, it leads to DNA damage, activation of the apoptotic pathway, and induction of transcriptome change that leads to generation of ROS. Dexrazoxane binds to both Top2a and Top2b, thereby preventing anthracycline binding of Top2 and consequent doxorubicin-induced cell death (29). This hypothesis is supported by the observation that ICRF 161, an analog of dexrazoxane with iron-chelating 
properties but lacking activity against Top2, is unable to confer cardioprotection in rat models (30).

\section{REDUCING THE RISK OF TOXICITY}

Other strategies to reduce cardiotoxicity have also been evaluated. Continuous infusion of doxorubicin is associated with less cardiac toxicity than bolus infusion $(31,32)$. However, this schedule is less convenient and hence is not a widely used strategy.

$4^{\prime}$-Epidoxorubicin, or epirubicin, is an analog of doxorubicin with equivalent efficacy and less cardiotoxicity on a milligram to milligram comparison (33-36). In a prospective randomized comparison of doxorubicin and epirubicin in patients with breast cancer, epirubicin was associated with a longer median duration of response at 11.9 months compared to 7.1 months with doxorubicin. The cumulative doses at which CHF occurred was $1134 \mathrm{mg} / \mathrm{m}^{2}$ with epirubicin compared to $492 \mathrm{mg} / \mathrm{m}^{2}$ with doxorubicin (15). In a meta-analysis of 13 studies comparing doxorubicin with epirubicin, the majority of which included women with advanced or metastatic breast cancer, epirubicin was associated with a significantly decreased risk of clinical cardiotoxicity, subclinical cardiotoxicity, and any cardiac event compared to doxorubicin (32). Epirubicin-induced cardiotoxicity is also dosedependent (37), and the United States Food and Drug Administration (FDA) recommends a maximum cumulative dose of $900 \mathrm{mg} / \mathrm{m}^{2}$. However, in an analysis of 1097 patients, the safe maximum cumulative dosage was found to be lower when risk factors such as age, radiation, and underlying cardiac risk factors were taken into consideration (38).

Altering the pharmacokinetic and pharmacodynamic profile of doxorubicin via liposome encapsulation has been an extremely effective strategy to minimize cardiotoxicity and maximize efficacy (39). One formulation of liposomal doxorubicin (Myocet) has polyethylene glycol embedded in the lipid layers, allowing the drug to stay in the liposome until it reaches the target tumor site (40). It is thought that the small size of the liposomes enables the drug to penetrate the compromised vasculature of tumors, allowing preferential delivery of the drug to the target tumor. Pegylation protects the liposome from uptake by the reticular-endothelial system, thereby increasing blood circulation time. Pegylated liposomal doxorubicin (PLD [Doxil/Caelyx]) has a half-life of $73.9 \mathrm{~h}$; this prolonged circulation time enables greater uptake of the drug by tumor. A phase III trial was conducted in 509 women with metastatic breast cancer to compare the efficacy and safety of PLD and conventional doxorubicin in the first-line setting. Response rates, progression-free survival (PFS), and OS were similar in both arms; however, overall risk of cardiotoxicity was significantly higher with conventional doxorubicin than with $\operatorname{PLD}(P<0.001, \mathrm{HR}=3.16)$ (41). In a meta-analysis of four studies comparing liposomal doxorubicin with conventional doxorubicin for the treatment of breast cancer or multiple myeloma, liposomal doxorubicin appeared to have similar efficacy to doxorubicin but was associated with significantly less risk of clinical or subclinical cardiotoxicity or any cardiotoxic event (32).

\section{TRASTUZUMAB-INDUCED CARDIOTOXICITY}

Approximately $20-25 \%$ of breast cancers overexpress the human epidermal growth factor receptor-2 (HER2 or ErbB2) (42, 43).
Such overexpression has been associated with more aggressive tumor biology, altered responsiveness to therapy, and poor clinical outcome including shortened survival (44). The development of trastuzumab, a humanized monoclonal antibody that targets the HER2 receptor, has been a major advance in the treatment of HER2-positive breast cancer.

The pivotal trial that led to the FDA approval of trastuzumab in 1998 enrolled 469 patients with previously untreated HER2positive metastatic breast cancer who were randomized to receive chemotherapy alone (consisting of an anthracycline plus cyclophosphamide in anthracycline-naïve patients or paclitaxel in patients previously treated with an anthracycline) or chemotherapy plus trastuzumab (45). The combination of chemotherapy plus trastuzumab resulted in an improved response rate (50 versus $32 \%$; $P<0.001$ ), TTP (median 7.4 versus 4.6 months; $P<0.001$ ), and $O S$ (median 25.1 versus 20.3 months; $P=0.046$ ) when compared with chemotherapy alone.

An unanticipated serious adverse event that emerged from the study was cardiac dysfunction. The rates of cardiac toxicity in patients randomized to doxorubicin and cyclophosphamide (AC) with trastuzumab versus AC alone were 27 and $8 \%$, respectively, and the rates of cardiac dysfunction in patients who received paclitaxel and trastuzumab versus paclitaxel alone were 13 and $1 \%$, respectively. The incidence of New York Heart Association (NYHA) class III or IV heart failure was highest among patients receiving AC plus trastuzumab: 16\%, compared with $3 \%$ for patients receiving AC alone, $2 \%$ for paclitaxel plus trastuzumab, and $1 \%$ for paclitaxel alone. Because the combination of trastuzumab plus doxorubicin resulted in unacceptably high rates of cardiac toxicity, these agents are generally not administered concurrently unless within the context of a clinical trial.

As a result of the above findings, the major adjuvant trials allowed only sequential administration of anthracyclines and trastuzumab (46-54). In addition, patients with abnormal cardiac function were excluded, the cumulative doxorubicin dose was limited to $300 \mathrm{mg} / \mathrm{m}^{2}$, and strict cardiac monitoring was mandated. The incidence of severe cardiotoxicity (NYHA class III or IV) in the adjuvant trials was modest: $0-4.1 \%$ in trastuzumabtreated patients versus $0-1.3 \%$ in the non-trastuzumab population (see Table 2). A meta-analysis of the five major adjuvant trials reported a 2.5-fold higher risk of cardiotoxicity with trastuzumab (54). Not surprisingly, a higher incidence of cardiotoxicity has been noted in patients also treated with anthracyclines and in patients who received a longer duration of trastuzumab in the adjuvant setting $(55,56)$.

Risk factors for the development of trastuzumab-related cardiotoxicity include prior or concurrent anthracycline use, age $>50$ years, pre-existing cardiac dysfunction, use of antihypertensive medication, and higher body mass index (56-59). The risk appears to be highest when trastuzumab is administered concurrently with anthracyclines, mostly when the cumulative dose of doxorubicin exceeds $300 \mathrm{mg} / \mathrm{m}^{2}$ (60). Cumulative doses up to $180 \mathrm{mg} / \mathrm{m}^{2}$ may in fact be safe to administer concurrently with trastuzumab (61), although there are no data demonstrating that such an approach would be more effective than sequential administration. 
Table 2 | Incidence of trastuzumab-associated cardiac events in adjuvant breast cancer trials.

\begin{tabular}{|c|c|c|c|c|}
\hline Trial & $\begin{array}{l}\text { Number of patients } \\
\text { in analysis }\end{array}$ & Treatment arm & $\begin{array}{l}\text { Incidence of } \\
\text { cardiac events (\%) }\end{array}$ & Definition of cardiac event \\
\hline NSABP B-31 $(46,51)$ & 814 & $A C \rightarrow P$ & 0.8 & $\begin{array}{l}\text { NYHA class III/IV CHF or } \\
\text { possible/probable cardiac death }\end{array}$ \\
\hline \multirow[t]{2}{*}{ NCCTG N9831 (52) } & 664 & $A C \rightarrow P$ & 0.3 & \multirow{2}{*}{$\begin{array}{l}\text { Symptomatic CHF or } \\
\text { probably/definite cardiac death }\end{array}$} \\
\hline & 710 & $\mathrm{AC} \rightarrow \mathrm{P} \rightarrow \mathrm{H}$ & 2.8 & \\
\hline \multirow[t]{3}{*}{ HERA (53) } & 1744 & Chemotherapy & 0.1 & \multirow{3}{*}{$\begin{array}{l}\text { NYHA class III/IV CHF with decrease } \\
\text { in LVEF } \geq 10 \% \text { from baseline to LVEF } \\
<50 \% \text {; or cardiac death }\end{array}$} \\
\hline & 1682 & Chemotherapy +1 year of Trastuzumab & 0.8 & \\
\hline & 1673 & Chemotherapy +2 years of Trastuzumab & 1.0 & \\
\hline \multirow[t]{2}{*}{ BCIRG 006 (48) } & 1073 & $\mathrm{AC} \rightarrow \mathrm{T}$ & 0.7 & \multirow[t]{2}{*}{ NYHA class III/IV CHF } \\
\hline & 1074 & $\mathrm{AC} \rightarrow \mathrm{TH}$ & 2 & \\
\hline
\end{tabular}

AC, doxorubicin + cyclophosphamide; P, paclitaxel; H, trastuzumab; T, docetaxel; $C$, carboplatin.

\section{MECHANISM OF TRASTUZUMAB-INDUCED CARDIOTOXICITY}

Unlike anthracycline-induced cardiotoxicity, trastuzumab-related cardiotoxicity is classified as Type II and is clinically and mechanistically distinct (62). Type II cardiotoxicity is not dose-dependent, is highly reversible, and is not associated with ultrastructural changes. This was demonstrated in a study of 38 patients with suspected trastuzumab-related cardiotoxicity, all of whom had received prior doxorubicin (63). Mean LVEF prior to initiation of trastuzumab was $61 \%$, decreased to $43 \%$ after trastuzumab, and increased to $56 \%$ after withdrawal of trastuzumab. Increases of LVEF were seen in 37 out of the 38 patients and mean time to recovery was 1.5 months. Six of the patients recovered without medical treatment. Nine patients underwent endomyocardial biopsy and the ultrastructural changes typical of anthracycline damage were not seen. The absence of such changes on biopsy demonstrates that the mechanism by which trastuzumab induces cardiac dysfunction is different from anthracyclines and is likely due to myocardial stunning or hibernation.

The pathogenesis of trastuzumab cardiotoxicity is not completely understood. The HER2 signaling pathway is essential for cardiac development and function (64), and mouse models lacking HER2 exhibit multiple characteristics of dilated cardiomyopathy, including chamber dilation, wall thinning, and decreased contractility (65). HER2 signaling is upregulated in animal models when the myocardium is under stress (66) Binding of trastuzumab to HER2 is thought to disrupt HER2-HER4 heterodimerization, thus disabling the protective mechanisms in the cardiac myocyte that are essential during exposure to adverse conditions or cardiac toxins (67). This hypothesis is consistent with the observation that increased cardiac toxicity is seen when trastuzumab is used in association with anthracyclines. Indeed, HER2-deficient cardiac myocytes are more susceptible to anthracycline-induced damage $(65,68)$. Activation of HER2 signaling by neuregulin-1 has been shown to improve cardiac myocyte function and survival, and upregulation of this pathway in the heart may be a potential therapeutic approach (69).

\section{CLINICAL USE OF TRASTUZUMAB}

The risk of cardiac toxicity with trastuzumab needs to be considered within the clinical context. In the metastatic setting, the benefits conferred by the addition of trastuzumab often outweigh the potential cardiac risks. On the other hand, in the early-stage setting, a significant number of patients will be cured with local therapy alone and may not in fact require adjuvant systemic therapy. However, since it is not currently possible to identify these patients, adjuvant therapy is offered to many who may not in fact derive benefit. One must therefore be especially judicious when selecting an adjuvant regimen, which may expose a patient to unnecessary toxicities, including CHF.

Because of the increased risks of cardiac toxicity seen with both the anthracyclines and trastuzumab, there has been much interest in the development of non-anthracycline-containing trastuzumab regimens. Of the pivotal adjuvant trastuzumab trials, Breast Cancer International Research Group (BCIRG) 006 was the only one to include a non-anthracycline-containing arm (48). In this study, 3222 patients with HER2+ breast cancer were randomized to receive AC followed by docetaxel (AC-T), AC-T plus trastuzumab initiated concurrently with docetaxel (AC-TH), or docetaxel + carboplatin + trastuzumab $(\mathrm{TCH})$. No significant differences in DFS and OS were found between the two trastuzumab regimens. However, there were significant differences in the incidence of CHF: $0.4 \%$ in the AC-T arm, $2.0 \%$ in the AC-TH arm, and $0.7 \%$ in the $\mathrm{TCH}$ arm. In addition, $\mathrm{AC}-\mathrm{TH}$ was associated with a significantly increased risk of persistent decline in EF at 4 years compared to TCH.

A recent retrospective population-based cohort study evaluated the long-term risk of heart failure (in this case defined as hospitalization or two ambulatory visits within 12 months) 
associated with adjuvant trastuzumab and chemotherapy (70). Women included in this study were diagnosed with early-stage breast cancer between 2003 and 2009. Those with metastatic breast cancer or a pre-existing diagnosis of heart failure were excluded. 19,074 women treated with chemotherapy were identified, of whom $18 \%$ also received adjuvant trastuzumab. After a median follow-up of 5.9 years, investigators found that adjuvant trastuzumab was associated with an increased risk of heart failure (5.3 versus $2.6 \%, P<0.0001)$. However, the increased risk was seen only within the first 1.5 years of treatment $(\mathrm{HR}=5.77,95 \%$ CI 4.38-7.62, $p=0.0004$ ); thereafter, there was no difference in the risk of developing CHF between women treated with trastuzumab plus chemotherapy and those treated with chemotherapy alone $(\mathrm{HR}=0.87,95 \%$ CI $0.57-1.33, p=0.53)$. These data are reassuring in that the risk of trastuzumab-associated cardiotoxicity appears to be limited to the period of active treatment.

No evidence-based guidelines exist for cardiac monitoring while on trastuzumab therapy. The FDA-approved manufacturer's package insert recommends that a baseline assessment of cardiac function be performed prior to the initiation of therapy and LVEF measurements should be repeated every 3 months during and upon completion of therapy (71). If trastuzumab is withheld for significant cardiac dysfunction, LVEF measurement should be repeated at 4 -week intervals. In the adjuvant setting it is recommended that LVEF be assessed every 6 months for at least 2 years following completion of therapy. In the metastatic setting, however, symptom-triggered evaluation of LVEF may be more appropriate given the risk-benefit ratio.

Trastuzumab should be held for 4 weeks if LVEF declines $\geq 16 \%$ from baseline or if there is $a \geq 10 \%$ decrease in LVEF from baseline to below the lower limit of normal. Treatment may be restarted if LVEF returns to normal within 4-8 weeks. Trastuzumab should be discontinued in the presence of symptomatic heart failure.

\section{CARDIOVASCULAR RISK WITH OTHER HER2-DIRECTED THERAPIES}

Lapatinib is a reversible small tyrosine kinase inhibitor that targets both the epidermal growth factor receptor (EGFR or HER1) and
HER2. It is indicated for use in combination with capecitabine for the treatment of metastatic HER2+ breast cancer after progression on an anthracycline, taxane and trastuzumab. In the phase III trial that led to its approval, asymptomatic declines in EF occurred in 4 of $164(2.4 \%)$ of patients receiving lapatinib plus capecitabine versus 1 of $152(0.7 \%)$ of patients receiving capecitabine alone. All four patients who experienced a cardiac event on lapatinib recovered and there were no symptomatic events (72) (see Table 3). It is important to note that the patients in this trial were highly selected as all had previously received trastuzumab and cardiac dysfunction was an exclusion criterion.

In a retrospective analysis of 3689 patients with various solid tumor types treated with lapatinib, only $1.6 \%$ of patients developed a cardiac event (73). Similar rates of cardiotoxicity were noted in patients who were pretreated with anthracyclines or trastuzumab compared to those who were not pretreated. Most of the cardiac events were usually asymptomatic and reversible, indicating a Type II cardiac toxicity. More recently, data were presented from the Adjuvant Lapatinib and/or Trastuzumab Treatment Optimization (ALTTO) trial in which patients with early-stage HER2+ breast cancer were randomized to receive adjuvant chemotherapy with trastuzumab, chemotherapy with concurrent trastuzumab and lapatinib, or chemotherapy with sequential trastuzumab and lapatinib (74). The incidence of NYHA Class III/IV heart failure was $<1 \%$ in all arms, with $97 \%$ of all patients having received an anthracycline.

Pertuzumab is a humanized monoclonal antibody that binds to HER at its dimerization subdomain. When combined with trastuzumab and docetaxel in the first-line setting for metastatic HER2+ breast cancer, there was an improvement in PFS and OS compared to trastuzumab and docetaxel (75). Interestingly, in a cardiac analysis of the trial, left ventricular dysfunction was numerically higher in the control arm (8.3 versus $4.4 \%$ for all grades). Declines in EF of at least $10 \%$ from baseline to $<50 \%$ occurred in 6.6 versus $3.8 \%$ of patients in the placebo and pertuzumab arms, respectively (76). Thus the combination of both antibodies did not increase the risk of cardiac adverse events.

Table 3 | Incidence of cardiac events with other HER2-directed therapies.

\begin{tabular}{|c|c|c|c|c|}
\hline Trial & $\begin{array}{l}\text { Number of patients } \\
\text { in analysis }\end{array}$ & HER2-directed therapy & $\begin{array}{l}\text { Incidence of } \\
\text { cardiac events (\%) }\end{array}$ & Definition of cardiac event \\
\hline \multirow[t]{2}{*}{ Geyer et al. (72) } & 161 & Capecitabine & 0.7 & Symptomatic decline in LVEF or \\
\hline & 163 & Lapatinib plus Capecitabine & 2.4 & $\begin{array}{l}\text { decrease } \geq 20 \% \text { from baseline to } \\
\text { below institution's lower limit of normal }\end{array}$ \\
\hline \multirow[t]{3}{*}{ ALTTO (74) } & 2097 & Trastuzumab alone & 0.86 & NYHA Class III/IV CHF or cardiac death \\
\hline & 2091 & Trastuzumab followed by Lapatinib & 0.25 & \\
\hline & 2093 & Trastuzumab concurrent with Lapatinib & 0.97 & \\
\hline \multirow[t]{2}{*}{ CLEOPATRA (76) } & 397 & Trastuzumab + docetaxel plus placebo & 6.6 & LVEF decline to $<50 \%$ with decrease \\
\hline & 407 & Trastuzumab + docetaxel plus Pertuzumab & 3.8 & $\geq 10 \%$ from baseline \\
\hline \multirow[t]{2}{*}{ EMILIA (77) } & 445 & Lapatinib + capecitabine & 1.6 & LVEF decline to $<50 \%$ with decrease \\
\hline & 481 & T-DM1 & 1.7 & $\geq 15 \%$ from baseline \\
\hline
\end{tabular}


Ado-trastuzumab emtansine (T-DM1) is an antibody-drug conjugate which combines trastuzumab with the cytoxic agent emtansine. The EMILIA trial randomized 991 patients with advanced breast cancer previously treated with trastuzumab and a taxane to TDM-1 versus lapatinib plus capecitabine. Results demonstrated an improvement in PFS and OS with T-DM1 compared to lapatinib plus capecitabine. Cardiotoxicity was $1.7 \%$ with T-DM1 versus $1.6 \%$ in the other arm (77).

\section{CARDIOVASCULAR RISK WITH BEVACIZUMAB}

In recent years, there has been considerable interest in the use of antiangiogenic agents for the treatment of various cancers. Bevacizumab, a monoclonal antibody targeting vascular endothelial growth factor (VEGF), was initially granted accelerated approval in 2008 for metastatic breast cancer based on impressive results in the first-line setting when combined with paclitaxel (78). However, while subsequent trials in the first-line metastatic setting confirmed a benefit in PFS, the magnitude of benefit was small, and no OS benefit was shown in any of the studies $(79,80)$. Three subsequent large adjuvant studies also failed to demonstrate a benefit in DFS or OS and consequently the FDA withdrew approval for bevacizumab in 2011. Nevertheless, as this agent is still used widely in other types of solid tumors, familiarly with its potential cardiac toxicities is important.

A meta-analysis of 5 randomized trials in metastatic breast cancer showed a statistically significant increased risk in grade 3 or higher hypertension ( 9.71 versus $0.64 \%, \mathrm{OR}=12.76)$ and left ventricular dysfunction ( 1.73 versus $0.78 \%, \mathrm{OR}=2.25$ ) in patients treated with bevacizumab compared to those who did not receive the drug (81). Similar increased risks were also seen in other studies, including the three adjuvant trials (82-84). The mechanism of bevacizumab-induced hypertension is not known but may be related to nitric oxide: VEGF is thought to increase production of nitric oxide, resulting in vasodilation (85). Inhibition of nitric oxide by bevacizumab therefore leads to vasoconstriction and consequent hypertension. The mechanism of CHF is also unclear, but the development of secondary hypertension itself could be a contributing factor.

\section{BIOMARKERS}

Current methods for detecting cardiotoxicity rely on evaluation of LVEF by either echocardiography or Multiple Gated Acquisition (MUGA) scan. However, by the time a decrease in EF is detected there has already been considerable myocardial damage. There is therefore a need to develop biomarkers that enable the early identification of cardiac deterioration. Such a strategy would allow for the implementation of preventive measures prior to the development of functional impairment.

Cardiac troponins I (cTnI) and T (cTnT) are sensitive and specific biomarkers of myocardial damage, whereas B-type natriuretic peptide (BNP) is a marker of volume overload. These markers have been studied as potential indicators of treatment-related cardiotoxicity.

In a study of 204 patients with a variety of malignancies receiving high-dose chemotherapy, investigators measured levels of cTnI in the plasma after every cycle. Patients were divided into troponin positive $(\mathrm{cTnI}+)$ and troponin negative $(\mathrm{cTnI}-)$ groups. In the cTnI- group, LVEF decreased transiently after chemotherapy, with a nadir after 3 months, but later recovered. On the other hand, patients in the cTnI+ group sustained greater reductions in LVEF, and the declines persisted even at the end of 7 months' follow-up (86). The same group of investigators also evaluated the significance of persistent elevations in cTnI+ and found that patients treated with high-dose chemotherapy who had persistent increases in cTn1 had a higher incidence of cardiac toxicity (85\%) compared to patients with transient increases (37\%) or who had no elevation in cTn1 (1\%) (87). Similarly, persistent proBNP elevation following chemotherapy has been associated with significantly lower LVEF (88). Given the heterogeneity of the study populations, however, definitive conclusions cannot be drawn at this time and more research is needed to determine whether these findings can be replicated.

\section{TREATMENT AND PREVENTION OF LV DYSFUNCTION}

Beta-blockers and angiotensin converting enzyme (ACE) inhibitors are standard treatments that have been shown to improve outcomes in patients with heart failure from a variety of etiologies. Treatment of cardiac dysfunction resulting from antineoplastic therapy follows the general cardiology guidelines for heart failure, although this practice seems to be based primarily on extrapolation rather than on evidence specifically addressing heart failure in the cancer population.

In a study of 201 consecutive patients with anthracyclineinduced CHF with LVEF $\leq 45 \%$, enalapril and, when tolerated, carvedilol were initiated as soon as LVEF impairment was detected (89). The investigators found that time elapsed from the end of chemotherapy to the start of heart failure therapy was a crucial variable for recovery of cardiac function. Among patients treated within 2 months after the end of chemotherapy, 64\% had a complete recovery of LVEF. After 2 months, however, the percentage of patients decreased, with no complete recovery seen after 6 months.

There has been significant interest evaluating the role of betablockers and ACE inhibitors as prophylactic agents in patients who are at risk of developing treatment-related cardiotoxicity. In a study that enrolled 50 patients receiving anthracycline therapy for a variety of cancers, patients were randomized to receive prophylactic carvedilol or placebo (90). Baseline LVEF was similar in both groups prior to initiation of chemotherapy. At 6 months of followup, one patient in the carvedilol group had $\mathrm{EF}<50 \%$ compared to five patients in the control group. The mean $\mathrm{EF}$ in the carvedilol group remained unchanged at the end of follow-up (70.5 versus $69.7 \%$ ) but was significantly lower in the control group (68.9 versus $52.3 \%$ ). However, there were some discrepancies in the reported anthracycline doses used in the study and caution should therefore be exercised when interpreting these data.

In a trial by Cardinale et al., 114 patients receiving high-dose chemotherapy and deemed at high risk for cardiotoxicity based on troponin I elevation were randomized to receive the ACE inhibitor enalapril or not (91). Enalapril was started 1 month after chemotherapy and continued for 1 year. The primary endpoint was an absolute decrease of $>10 \%$ in LVEF, with a decline below the lower limit of normal. Forty-three percent of patients in 
the control arm met the primary endpoint, compared to none in the treatment group $(P<0.001)$. These results suggest that in patients with evidence of early cardiac damage as measured by elevated troponin I values, early institution of ACE inhibitor therapy may prevent the progression of cardiac toxicity. The prevention of cardiac dysfunction during adjuvant breast cancer therapy (PRADA) trial is an ongoing randomized, placebo-controlled study evaluating the role of beta-blockers and ACE inhibitors in preventing cardiac dysfunction during adjuvant breast cancer therapy (92).

\section{LACK OF CLINICAL GUIDELINES}

Unfortunately, the American College of Cardiology and the American Heart Association have not provided any guidelines for the detection, prevention, monitoring, or treatment of cardiotoxicity from antineoplastic therapy. All patients receiving potentially cardiotoxic anticancer drugs are considered candidates for Stage A heart failure, meaning they are at increased risk of developing cardiac dysfunction (93). The European Society of Medical Oncology has published a comprehensive set of clinical practice guidelines for the management of patients with cardiotoxicity from chemotherapy, targeted agents, and radiation (94). A similar consensus statement from the major American cardiology societies would be extremely helpful to practicing clinicians to ensure maximal anticancer benefits from therapy with minimal cardiac complications.

\section{CONCLUSION}

While much progress has been made in the treatment of breast cancer, cardiac complications resulting from therapy remain a significant concern. Both anthracyclines and novel targeted agents can inflict cardiac damage, although the mechanisms by which they do so and their clinical manifestations appear to be distinct. The challenge for the future will be to develop methods for early detection of cardiac dysfunction, identify strategies for prevention and treatment of cardiotoxicity, and establish clinical guidelines for practicing physicians. Many questions remain unanswered, and ongoing research and collaboration between oncologists and cardiologists are needed to ensure optimal efficacy and safety of current and future anticancer agents.

\section{REFERENCES}

1. American Cancer Society. Cancer Treatment and Survivorship Facts and Figures, 2014-2015. Atlanta: American Cancer Society (2014).

2. Polychemotherapy for early breast cancer: an overview of the randomised trials. Early breast cancer trialists' collaborative group. Lancet (1998) 352(9132):930-42. doi:10.1016/S0140-6736(98)03301-7

3. A'Hern RP, Smith IE, Ebbs SR. Chemotherapy and survival in advanced breast cancer: the inclusion of doxorubicin in Cooper type regimens. BrJCancer (1993) 67(4):801-5. doi:10.1038/bjc.1993.146

4. Von Hoff DD, Layard MW, Basa P, Davis HL Jr, Von Hoff AL, Rozencweig M, et al. Risk factors for doxorubicin-induced congestive heart failure. Ann Intern Med (1979) 91(5):710-7. doi:10.7326/0003-4819-91-5-710

5. Lefrak EA, Pitha J, Rosenheim S, Gottlieb JA. A clinicopathologic analysis of adriamycin cardiotoxicity. Cancer (1973) 32(2):302-14. doi:10.1002/10970142(197308)32:2<302::AID-CNCR2820320205>3.0.CO;2-2

6. Steinherz LJ, Steinherz PG, Tan CT, Heller G, Murphy ML. Cardiac toxicity 4 to 20 years after completing anthracycline therapy. JAMA (1991) 266(12):1672-7. doi:10.1001/jama.266.12.1672
7. Kremer LC, van Dalen EC, Offringa M, Ottenkamp J, Voute PA. Anthracyclineinduced clinical heart failure in a cohort of 607 children: long-term follow-up study. J Clin Oncol (2001) 19(1):191-6.

8. Billingham ME, Mason JW, Bristow MR, Daniels JR. Anthracycline cardiomyopathy monitored by morphologic changes. Cancer Treat Rep (1978) 62(6):865-72.

9. Mackay B, Ewer MS, Carrasco CH, Benjamin RS. Assessment of anthracycline cardiomyopathy by endomyocardial biopsy. Ultrastruct Pathol (1994) 18(12):203-11. doi:10.3109/01913129409016291

10. Swain SM, Whaley FS, Ewer MS. Congestive heart failure in patients treated with doxorubicin: a retrospective analysis of three trials. Cancer (2003) 97(11):2869-79. doi:10.1002/cncr.11407

11. Lotrionte M, Biondi-Zoccai G, Abbate A, Lanzetta G, D’Ascenzo F, Malavasi V, et al. Review and meta-analysis of incidence and clinical predictors of anthracycline cardiotoxicity. Am J Cardiol (2013) 112(12):1980-4. doi:10.1016/j.amjcard. 2013.08.026

12. Doyle JJ, Neugut AI, Jacobson JS, Grann VR, Hershman DL. Chemotherapy and cardiotoxicity in older breast cancer patients: a population-based study. J Clin Oncol (2005) 23(34):8597-605. doi:10.1200/JCO.2005.02.5841

13. Shapiro CL, Hardenbergh PH, Gelman R, Blanks D, Hauptman P, Recht A, et al. Cardiac effects of adjuvant doxorubicin and radiation therapy in breast cancer patients. J Clin Oncol (1998) 16(11):3493-501.

14. Visscher H, Ross CJ, Rassekh SR, Barhdadi A, Dube MP, Al-Saloos H, et al. Pharmacogenomic prediction of anthracycline-induced cardiotoxicity in children. $J$ Clin Oncol (2012) 30(13):1422-8. doi:10.1200/JCO.2010.34.3467

15. Blanco JG, Sun CL, Landier W, Chen L, Esparza-Duran D, Leisenring W, et al. Anthracycline-related cardiomyopathy after childhood cancer: role of polymorphisms in carbonyl reductase genes - a report from the Children's Oncology Group. J Clin Oncol (2012) 30(13):1415-21. doi:10.1200/JCO.2011.34.8987

16. Myers C, Bonow R, Palmeri S, Jenkins J, Corden B, Locker G, et al. A randomized controlled trial assessing the prevention of doxorubicin cardiomyopathy by N-acetylcysteine. Semin Oncol (1983) 10(1 Suppl 1):53-5.

17. Ladas EJ, Jacobson JS, Kennedy DD, Teel K, Fleischauer A, Kelly KM. Antioxidants and cancer therapy: a systematic review. J Clin Oncol (2004) 22(3):517-28. doi:10.1200/JCO.2004.03.086

18. Simunek T, Sterba M, Popelova O, Adamcova M, Hrdina R, Gersl V. Anthracycline-induced cardiotoxicity: overview of studies examining the roles of oxidative stress and free cellular iron. Pharmacol Rep (2009) 61(1):154-71. doi:10.1016/S1734-1140(09)70018-0

19. Hasinoff BB, Patel D, Wu X. The oral iron chelator ICL670A (deferasirox) does not protect myocytes against doxorubicin. Free Radic Biol Med (2003) 35(11):1469-79. doi:10.1016/j.freeradbiomed.2003.08.005

20. Zhang S, Liu X, Bawa-Khalfe T, Lu LS, Lyu YL, Liu LF, et al. Identification of the molecular basis of doxorubicin-induced cardiotoxicity. Nat Med (2012) 18(11):1639-42. doi:10.1038/nm.2919

21. Speyer JL, Green MD, Zeleniuch-Jacquotte A, Wernz JC, Rey M, Sanger J, et al. ICRF-187 permits longer treatment with doxorubicin in women with breast cancer. J Clin Oncol (1992) 10(1):117-27.

22. Swain SM, Whaley FS, Gerber MC, Ewer MS, Bianchine JR, Gams RA. Delayed administration of dexrazoxane provides cardioprotection for patients with advanced breast cancer treated with doxorubicin-containing therapy. J Clin Oncol (1997) 15(4):1333-40.

23. Swain SM, Whaley FS, Gerber MC, Weisberg S, York M, Spicer D, et al. Cardioprotection with dexrazoxane for doxorubicin-containing therapy in advanced breast cancer. J Clin Oncol (1997) 15(4):1318-32.

24. Marty M, Espie M, Llombart A, Monnier A, Rapoport BL, Stahalova V. Multicenter randomized phase III study of the cardioprotective effect of dexrazoxane (Cardioxane) in advanced/metastatic breast cancer patients treated with anthracycline-based chemotherapy. Ann Oncol (2006) 17(4):614-22. doi:10. 1093/annonc/mdj134

25. Venturini M, Michelotti A, Del Mastro L, Gallo L, Carnino F, Garrone O, et al. Multicenter randomized controlled clinical trial to evaluate cardioprotection of dexrazoxane versus no cardioprotection in women receiving epirubicin chemotherapy for advanced breast cancer. J Clin Oncol (1996) 14(12):3112-20.

26. Seymour L, Bramwell V, Moran LA. Use of dexrazoxane as a cardioprotectant in patients receiving doxorubicin or epirubicin chemotherapy for the treatment of cancer. The provincial systemic treatment disease site group. Cancer Prev Control (1999) 3(2):145-59. 
27. Hasinoff BB. The interaction of the cardioprotective agent ICRF-187 [+)1,2-bis(3,5-dioxopiperazinyl-1-yL)propane); its hydrolysis product (ICRF-198); and other chelating agents with the $\mathrm{Fe}$ (III) and $\mathrm{Cu}$ (II) complexes of adriamycin. Agents Actions (1989) 26(3-4):378-85. doi:10.1007/BF01967305

28. Hasinoff BB, Kala SV. The removal of metal ions from transferrin, ferritin and ceruloplasmin by the cardioprotective agent ICRF-187 [(+)-1,2-bis(3,5dioxopiperazinyl-1-yl)propane] and its hydrolysis product ADR-925. Agents Actions (1993) 39(1-2):72-81. doi:10.1007/BF01975717

29. Vejpongsa P, Yeh ET. Topoisomerase 2beta: a promising molecular target for primary prevention of anthracycline-induced cardiotoxicity. Clin Pharmacol Ther (2014) 95(1):45-52. doi:10.1038/clpt.2013.201

30. Martin E, Thougaard AV, Grauslund M, Jensen PB, Bjorkling F, Hasinoff BB, et al. Evaluation of the topoisomerase II-inactive bisdioxopiperazine ICRF-161 as a protectant against doxorubicin-induced cardiomyopathy. Toxicology (2009) 255(1-2):72-9. doi:10.1016/j.tox.2008.10.011

31. Hortobagyi GN, Frye D, Buzdar AU, Ewer MS, Fraschini G, Hug V, et al. Decreased cardiac toxicity of doxorubicin administered by continuous intravenous infusion in combination chemotherapy for metastatic breast carcinoma. Cancer (1989) 63(1):37-45. doi:10.1002/1097-0142(19890101)63:1<37::AIDCNCR2820630106>3.0.CO;2-Z

32. Smith LA, Cornelius VR, Plummer CJ, Levitt G, Verrill M, Canney P, et al. Cardiotoxicity of anthracycline agents for the treatment of cancer: systematic review and meta-analysis of randomised controlled trials. BMC Cancer (2010) 10:337. doi:10.1186/1471-2407-10-337

33. Brambilla C, Rossi A, Bonfante V, Ferrari L, Villani F, Crippa F, et al. Phase II study of doxorubicin versus epirubicin in advanced breast cancer. Cancer Treat Rep (1986) 70(2):261-6.

34. Jain KK, Casper ES, Geller NL, Hakes TB, Kaufman RJ, Currie V, et al. A prospective randomized comparison of epirubicin and doxorubicin in patients with advanced breast cancer. J Clin Oncol (1985) 3(6):818-26.

35. Kaklamani VG, Gradishar WJ. Epirubicin versus doxorubicin: which is the anthracycline of choice for the treatment of breast cancer? Clin Breast Cancer (2003) 4(Suppl 1):S26-33. doi:10.3816/CBC.2003.s.012

36. Leyvraz S, Bacchi M, Cerny T, Lissoni A, Sessa C, Bressoud A, et al. Phase I multicenter study of combined high-dose ifosfamide and doxorubicin in the treatment of advanced sarcomas. Swiss Group for Clinical Research (SAKK). Ann Oncol (1998) 9(8):877-84. doi:10.1023/A:1008464504583

37. Ryberg M, Nielsen D, Skovsgaard T, Hansen J, Jensen BV, Dombernowsky P. Epirubicin cardiotoxicity: an analysis of 469 patients with metastatic breast cancer. J Clin Oncol (1998) 16(11):3502-8.

38. Ryberg M, Nielsen D, Cortese G, Nielsen G, Skovsgaard T, Andersen PK. New insight into epirubicin cardiac toxicity: competing risks analysis of 1097 breast cancer patients. J Natl Cancer Inst (2008) 100(15):1058-67. doi:10.1093/jnci/ djn206

39. Robert NJ, Vogel CL, Henderson IC, Sparano JA, Moore MR, Silverman P, et al. The role of the liposomal anthracyclines and other systemic therapies in the management of advanced breast cancer. Semin Oncol (2004) 31(6 Suppl 13):106-46. doi:10.1053/j.seminoncol.2004.09.018

40. Swenson CE, Bolcsak LE, Batist G, Guthrie TH Jr, Tkaczuk KH, Boxenbaum $\mathrm{H}$, et al. Pharmacokinetics of doxorubicin administered i.v. as Myocet (TLC D-99; liposome-encapsulated doxorubicin citrate) compared with conventional doxorubicin when given in combination with cyclophosphamide in patients with metastatic breast cancer. Anticancer Drugs (2003) 14(3):239-46. doi:10.1097/00001813-200303000-00008

41. O’Brien ME, Wigler N, Inbar M, Rosso R, Grischke E, Santoro A, et al. Reduced cardiotoxicity and comparable efficacy in a phase III trial of pegylated liposomal doxorubicin $\mathrm{HCl}$ (CAELYX/Doxil) versus conventional doxorubicin for first-line treatment of metastatic breast cancer. Ann Oncol (2004) 15(3):440-9. doi:10.1093/annonc/mdh097

42. Slamon DJ, Clark GM, Wong SG, Levin WJ, Ullrich A, McGuire WL. Human breast cancer: correlation of relapse and survival with amplification of the HER-2/neu oncogene. Science (1987) 235(4785):177-82. doi:10.1126/science. 3798106

43. Owens MA, Horten BC, Da Silva MM. HER2 amplification ratios by fluorescence in situ hybridization and correlation with immunohistochemistry in a cohort of 6556 breast cancer tissues. Clin Breast Cancer (2004) 5(1):63-9. doi:10.3816/CBC.2004.n.011
44. Kallioniemi OP, Holli K, Visakorpi T, Koivula T, Helin HH, Isola JJ. Association of c-erbB-2 protein over-expression with high rate of cell proliferation, increased risk of visceral metastasis and poor long-term survival in breast cancer. Int $J$ Cancer (1991) 49(5):650-5. doi:10.1002/ijc.2910490504

45. Slamon DJ, Leyland-Jones B, Shak S, Fuchs H, Paton V, Bajamonde A, et al. Use of chemotherapy plus a monoclonal antibody against HER2 for metastatic breast cancer that overexpresses HER2. N Engl J Med (2001) 344(11):783-92. doi:10.1056/NEJM200103153441101

46. Romond EH, Perez EA, Bryant J, Suman VJ, Geyer CE Jr, Davidson NE, et al. Trastuzumab plus adjuvant chemotherapy for operable HER2-positive breast cancer. N Engl J Med (2005) 353(16):1673-84. doi:10.1056/NEJMoa052122

47. Piccart-Gebhart MJ, Procter M, Leyland-Jones B, Goldhirsch A, Untch M, Smith I, et al. Trastuzumab after adjuvant chemotherapy in HER2-positive breast cancer. N Engl J Med (2005) 353(16):1659-72. doi:10.1056/NEJMoa052306

48. Slamon D, Eiermann W, Robert N, Pienkowski T, Martin M, Press M, et al. Adjuvant trastuzumab in HER2-positive breast cancer. N Engl J Med (2011) 365(14):1273-83. doi:10.1056/NEJMoa0910383

49. Perez EA, Romond EH, Suman VJ, Jeong JH, Davidson NE, Geyer CE Jr., et al. Four-year follow-up of trastuzumab plus adjuvant chemotherapy for operable human epidermal growth factor receptor 2-positive breast cancer: joint analysis of data from NCCTG N9831 and NSABP B-31. J Clin Oncol (2011) 29(25):3366-73. doi:10.1200/JCO.2011.35.0868

50. Joensuu H, Kellokumpu-Lehtinen PL, Bono P, Alanko T, Kataja V, Asola R, et al. Adjuvant docetaxel or vinorelbine with or without trastuzumab for breast cancer. N Engl J Med (2006) 354(8):809-20. doi:10.1056/NEJMoa053028

51. Tan-Chiu E, Yothers G, Romond E, Geyer CE Jr, Ewer M, Keefe D, et al. Assessment of cardiac dysfunction in a randomized trial comparing doxorubicin and cyclophosphamide followed by paclitaxel, with or without trastuzumab as adjuvant therapy in node-positive, human epidermal growth factor receptor 2overexpressing breast cancer: NSABP B-31. J Clin Oncol (2005) 23(31):7811-9. doi:10.1200/jco.2005.02.4091

52. Perez EA, Suman VJ, Davidson NE, Sledge GW, Kaufman PA, Hudis CA, et al. Cardiac safety analysis of doxorubicin and cyclophosphamide followed by paclitaxel with or without trastuzumab in the North Central Cancer Treatment Group N9831 adjuvant breast cancer trial. J Clin Oncol (2008) 26(8):1231-8. doi:10.1200/JCO.2007.13.5467

53. Goldhirsch A, Gelber RD, Piccart-Gebhart MJ, de Azambuja E, Procter M, Suter TM, et al. 2 years versus 1 year of adjuvant trastuzumab for HER2-positive breast cancer (HERA): an open-label, randomised controlled trial. Lancet (2013) 382(9897):1021-8. doi:10.1016/S0140-6736(13)61094-6

54. Viani GA, Afonso SL, Stefano EJ, De Fendi LI, Soares FV. Adjuvant trastuzumab in the treatment of her-2-positive early breast cancer: a meta-analysis of published randomized trials. BMC Cancer (2007) 7:153. doi:10.1186/14712407-7-153

55. Moja L, Tagliabue L, Balduzzi S, Parmelli E, Pistotti V, Guarneri V, et al. Trastuzumab containing regimens for early breast cancer. Cochrane Database Syst Rev (2012) 4:CD006243. doi:10.1002/14651858.CD006243.pub2

56. de Azambuja E, Procter MJ, van Veldhuisen DJ, Agbor-Tarh D, Metzger-Filho O, Steinseifer J, et al. Trastuzumab-associated cardiac events at 8 years of median follow-up in the herceptin adjuvant trial (BIG 1-01). J Clin Oncol (2014) 32(20):2159-65. doi:10.1200/JCO.2013.53.9288

57. Russell SD, Blackwell KL, Lawrence J, Pippen JE Jr, Roe MT, Wood F, et al. Independent adjudication of symptomatic heart failure with the use of doxorubicin and cyclophosphamide followed by trastuzumab adjuvant therapy: a combined review of cardiac data from the National surgical adjuvant breast and bowel project B-31 and the North Central Cancer Treatment Group N9831 clinical trials. J Clin Oncol (2010) 28(21):3416-21. doi:10.1200/JCO.2009.23.6950

58. Bowles EJ, Wellman R, Feigelson HS, Onitilo AA, Freedman AN, Delate T, et al. Risk of heart failure in breast cancer patients after anthracycline and trastuzumab treatment: a retrospective cohort study. J Natl Cancer Inst (2012) 104(17):1293-305. doi:10.1093/jnci/djs317

59. Romond EH, Jeong JH, Rastogi P, Swain SM, Geyer CE Jr, Ewer MS, et al. Seven-year follow-up assessment of cardiac function in NSABP B-31, a randomized trial comparing doxorubicin and cyclophosphamide followed by paclitaxel (ACP) with ACP plus trastuzumab as adjuvant therapy for patients with nodepositive, human epidermal growth factor receptor 2-positive breast cancer. J Clin Oncol (2012) 30(31):3792-9. doi:10.1200/JCO.2011.40.0010 
60. Seidman A, Hudis C, Pierri MK, Shak S, Paton V, Ashby M, et al. Cardiac dysfunction in the trastuzumab clinical trials experience. J Clin Oncol (2002) 20(5):1215-21. doi:10.1200/JCO.20.5.1215

61. Gianni L, Eiermann W, Semiglazov V, Manikhas A, Lluch A, Tjulandin S, et al. Neoadjuvant chemotherapy with trastuzumab followed by adjuvant trastuzumab versus neoadjuvant chemotherapy alone, in patients with HER2positive locally advanced breast cancer (the NOAH trial): a randomised controlled superiority trial with a parallel HER2-negative cohort. Lancet (2010) 375(9712):377-84. doi:10.1016/S0140-6736(09)61964-4

62. Ewer MS, Lippman SM. Type II chemotherapy-related cardiac dysfunction: time to recognize a new entity. J Clin Oncol (2005) 23(13):2900-2. doi:10.1200/JCO. 2005.05.827

63. Ewer MS, Vooletich MT, Durand JB, Woods ML, Davis JR, Valero V, et al. Reversibility of trastuzumab-related cardiotoxicity: new insights based on clinical course and response to medical treatment. J Clin Oncol (2005) 23(31):7820-6. doi:10.1200/JCO.2005.13.300

64. Kuramochi Y, Guo X, Sawyer DB. Neuregulin activates erbB2-dependent src/FAK signaling and cytoskeletal remodeling in isolated adult rat cardiac myocytes. J Mol Cell Cardiol (2006) 41(2):228-35. doi:10.1016/j.yjmcc.2006. 04.007

65. Crone SA, Zhao YY, Fan L, Gu Y, Minamisawa S, Liu Y, et al. ErbB2 is essential in the prevention of dilated cardiomyopathy. Nat Med (2002) 8(5):459-65. doi:10.1038/nm0502-459

66. Gabrielson K, Bedja D, Pin S, Tsao A, Gama L, Yuan B, et al. Heat shock protein 90 and ErbB2 in the cardiac response to doxorubicin injury. Cancer Res (2007) 67(4):1436-41. doi:10.1158/0008-5472.CAN-06-3721

67. De Keulenaer GW, Doggen K, Lemmens K. The vulnerability of the heart as a pluricellular paracrine organ: lessons from unexpected triggers of heart failure in targeted ErbB2 anticancer therapy. Circ Res (2010) 106(1):35-46. doi:10.1161/CIRCRESAHA.109.205906

68. Negro A, Brar BK, Lee KF. Essential roles of Her2/erbB2 in cardiac development and function. Recent Prog Horm Res (2004) 59:1-12. doi:10.1210/rp.59.1.1

69. Lemmens K, Doggen K, De Keulenaer GW. Role of neuregulin-1/ErbB signaling in cardiovascular physiology and disease: implications for therapy of heart failure. Circulation (2007) 116(8):954-60. doi:10.1161/CIRCULATIONAHA.107. 690487

70. Goldhar HA, Yan A, Ko D, Earle C, Tomlinson GA, Trudeau M, et al. Longterm risk of heart failure associated with adjuvant trastuzumab in breast cancer patients. J Clin Oncol (2014) 32(15s):abstr9504.

71. Herceptin (R) [package insert]. San Francisco, CA: Genentech, Inc (2014).

72. Geyer CE, Forster J, Lindquist D, Chan S, Romieu CG, Pienkowski T, et al. Lapatinib plus capecitabine for HER2-positive advanced breast cancer. N Engl J Med (2006) 355(26):2733-43. doi:10.1056/NEJMoa064320

73. Perez EA, Koehler M, Byrne J, Preston AJ, Rappold E, Ewer MS. Cardiac safety of lapatinib: pooled analysis of 3689 patients enrolled in clinical trials. Mayo Clin Proc (2008) 83(6):679-86. doi:10.4065/83.6.679

74. Piccart-Gebhart MJ, Holmes AP, Baselga J, De Azambuja E, Dueck AC, Viale G, et al. First results from the phase III ALTTO trial (BIG 2-06; NCCTG [Alliance] N063D) comparing one year of anti-HER2 therapy with lapatinib alone (L), trastuzumab alone $(\mathrm{T})$, their sequence $(\mathrm{T} \rightarrow \mathrm{L})$, or their combination $(\mathrm{T}+\mathrm{L})$ in the adjuvant treatment of HER2-positive early breast cancer (EBC). J Clin Oncol (2014) 32(18s):abstrLBA4.

75. Swain SM, Kim SB, Cortes J, Ro J, Semiglazov V, Campone M, et al. Pertuzumab, trastuzumab, and docetaxel for HER2-positive metastatic breast cancer (CLEOPATRA study): overall survival results from a randomised, doubleblind, placebo-controlled, phase 3 study. Lancet Oncol (2013) 14(6):461-71. doi:10.1016/S1470-2045(13)70130-X

76. Swain SM, Ewer MS, Cortes J, Amadori D, Miles D, Knott A, et al. Cardiac tolerability of pertuzumab plus trastuzumab plus docetaxel in patients with HER2-positive metastatic breast cancer in CLEOPATRA: a randomized, doubleblind, placebo-controlled phase III study. Oncologist (2013) 18(3):257-64. doi:10.1634/theoncologist.2012-0448

77. Verma S, Miles D, Gianni L, Krop IE, Welslau M, Baselga J, et al. Trastuzumab emtansine for HER2-positive advanced breast cancer. N Engl J Med (2012) 367(19):1783-91. doi:10.1056/NEJMoa1209124

78. Miller KD. E2100: a phase III trial of paclitaxel versus paclitaxel/bevacizumab for metastatic breast cancer. Clin Breast Cancer (2003) 3(6):421-2. doi:10.3816/ CBC.2003.n.007
79. Miles DW, Chan A, Dirix LY, Cortes J, Pivot X, Tomczak P, et al. Phase III study of bevacizumab plus docetaxel compared with placebo plus docetaxel for the first-line treatment of human epidermal growth factor receptor 2-negative metastatic breast cancer. J Clin Oncol (2010) 28(20):3239-47. doi:10.1200/JCO.2008.21.6457

80. Robert NJ, Dieras V, Glaspy J, Brufsky AM, Bondarenko I, Lipatov ON, et al. RIBBON-1: randomized, double-blind, placebo-controlled, phase III trial of chemotherapy with or without bevacizumab for first-line treatment of human epidermal growth factor receptor 2-negative, locally recurrent or metastatic breast cancer. J Clin Oncol (2011) 29(10):1252-60. doi:10.1200/JCO.2010.28. 0982

81. Cortes J, Calvo V, Ramirez-Merino N, O’Shaughnessy J, Brufsky A, Robert N, et al. Adverse events risk associated with bevacizumab addition to breast cancer chemotherapy: a meta-analysis. Ann Oncol (2012) 23(5):1130-7. doi:10.1093/ annonc/mdr432

82. Cameron D, Brown J, Dent R, Jackisch C, Mackey J, Pivot X, et al. Adjuvant bevacizumab-containing therapy in triple-negative breast cancer (BEATRICE): primary results of a randomised, phase 3 trial. Lancet Oncol (2013) 14(10):933-42. doi:10.1016/S1470-2045(13)70335-8

83. Slamon D, Swain S, Buyse MMM, Geyer CE, Im YH, et al. Primary results from BETH, a phase 3 controlled study of adjuvant chemotherapy and trastuzumab \pm bevacizumab in patients with HER2-positive, node-positive, or high-risk node-negative breast cancer. 2013 San Antonio Breast Cancer Symposium. Abstract S1-03 Cancer Res (2013) 73:S1-3. doi:10.1158/0008-5472. SABCS13-S1-03

84. Miller K, O’Neill A, Dang C, Northfelt DW, Gradishar WJ, Goldstein L. Bevacizumab $(\mathrm{Bv})$ in the adjuvant treatment of HER2-negative breast cancer: Final results from Eastern cooperative oncology group E5103. J Clin Oncol (2014) 32(15s):abstr500.

85. Hood JD, Meininger CJ, Ziche M, Granger HJ. VEGF upregulates ecNOS message, protein, and NO production in human endothelial cells. Am J Physiol (1998) 274(3 Pt 2):H1054-8.

86. Cardinale D, Sandri MT, Martinoni A, Tricca A, Civelli M, Lamantia G, et al. Left ventricular dysfunction predicted by early troponin I release after highdose chemotherapy. J Am Coll Cardiol (2000) 36(2):517-22. doi:10.1016/S07351097(00)00748-8

87. Cardinale D, Sandri MT, Colombo A, Colombo N, Boeri M, Lamantia G, et al. Prognostic value of troponin I in cardiac risk stratification of cancer patients undergoing high-dose chemotherapy. Circulation (2004) 109(22):2749-54. doi:10.1161/01.CIR.0000130926.51766.CC

88. Sandri MT, Salvatici M, Cardinale D, Zorzino L, Passerini R, Lentati P, et al. N-terminal pro-B-type natriuretic peptide after high-dose chemotherapy: a marker predictive of cardiac dysfunction? Clin Chem (2005) 51(8):1405-10. doi:10.1373/clinchem.2005.050153

89. Cardinale D, Colombo A, Lamantia G, Colombo N, Civelli M, De Giacomi G, et al. Anthracycline-induced cardiomyopathy: clinical relevance and response to pharmacologic therapy. J Am Coll Cardiol (2010) 55(3):213-20. doi:10.1016/j.jacc.2009.03.095

90. Kalay N, Basar E, Ozdogru I, Er O, Cetinkaya Y, Dogan A, et al. Protective effects of carvedilol against anthracycline-induced cardiomyopathy. J Am Coll Cardiol (2006) 48(11):2258-62. doi:10.1016/j.jacc.2006.07.052

91. Cardinale D, Colombo A, Sandri MT, Lamantia G, Colombo N, Civelli M, et al. Prevention of high-dose chemotherapy-induced cardiotoxicity in highrisk patients by angiotensin-converting enzyme inhibition. Circulation (2006) 114(23):2474-81. doi:10.1161/CIRCULATIONAHA.106.635144

92. Gulati G, Heck S, Ree AH, Bratland A, Stein K, Schulz-Menger J et al. Prevention of cardiac dysfunction during adjuvant breast cancer therapy: the PRADA study. Cancer Res (2014) 74(19s):CT321. doi:10.1158/1538-7445. am2014-ct321

93. O'Gara PT, Kushner FG, Ascheim DD, Casey DE Jr, Chung MK, de Lemos JA, et al. 2013 ACCF/AHA guideline for the management of heart failure. A report of the American college of cardiology foundation/American heart association task force on practice guidelines. Circulation (2013) 128:e240-327. doi:10.1161/CIR.0b013e31829e8776

94. Curigliano G, Cardinale D, Suter T, Plataniotis G, de Azambuja E, Sandri MT, et al. Cardiovascular toxicity induced by chemotherapy, targeted agents and radiotherapy: ESMO Clinical Practice Guidelines. Ann Oncol (2012) 23(Suppl 7):vii155-66. doi:10.1093/annonc/mds293 
95. Joensuu H, Bono P, Kataja V, Alanko T, Kokko R, Asola R, et al. Fluorouracil, epirubicin, and cyclophosphamide with either docetaxel or vinorelbine, with or without trastuzumab, as adjuvant treatments of breast cancer: final results of the FinHer Trial. J Clin Oncol (2009) 27(34):5685-92. doi:10.1200/JCO.2008.21.4577

Conflict of Interest Statement: The authors declare that the research was conducted in the absence of any commercial or financial relationships that could be construed as a potential conflict of interest. The Guest Associate Editor Sharad Goyal declares that, despite being affiliated to the same institution as author Shuang Guo \& Serena Wong, the review process was handled objectively and no conflict of interest exists.
Received: 16 September 2014; paper pending published: 09 October 2014; accepted: 18 November 2014; published online: 04 December 2014.

Citation: Guo S and Wong S (2014) Cardiovascular toxicities from systemic breast cancer therapy. Front. Oncol. 4:346. doi: 10.3389/fonc.2014.00346

This article was submitted to Radiation Oncology, a section of the journal Frontiers in Oncology.

Copyright (c) 2014 Guo and Wong. This is an open-access article distributed under the terms of the Creative Commons Attribution License (CC BY). The use, distribution or reproduction in other forums is permitted, provided the original author(s) or licensor are credited and that the original publication in this journal is cited, in accordance with accepted academic practice. No use, distribution or reproduction is permitted which does not comply with these terms. 